\title{
Tarifautonomie aus ökonomischer, juristischer und gesellschaftstheoretischer Sicht
}

Seitdem die Arbeitslosigkeit Mitte der 1990er Jahre drastisch angestiegen ist, richten sich Reformüberlegungen zur rechtlichen Gestaltung der Arbeitsbeziehungen wieder besonders auf die Tarifautonomie. Hohe Lohnabschlüsse gelten als beschäftigungsschädlich. $\mathrm{Ob}$ das zutrifft, soll hier nicht im Vordergrund stehen. Behandelt wird vielmehr die Frage, ob dies ein Grund sein kann, die Tarifautonomie zu beschneiden. Die Rechtswissenschaft hat für ihre Positionsfindung vor allem wirtschaftswissenschaftliche Studien aufgegriffen. Studien der Gesellschaftstheorie fanden kaum Beachtung - obwohl sie wichtige Aussagen zu kollektiver Autonomie treffen. Welche Folgen hatte die starke Beachtung ökonomischer Argumente? Und was kann die Gesellschaftstheorie zur rechtlichen Beurteilung der Tarifautonomie beitragen?

\section{Einleitung}

Aus wirtschaftswissenschaftlichen Studien stammende Argumente haben die Tarifautonomie unter enormen Rechtfertigungsdruck gesetzt. Die Tarifautonomie einzuschränken und damit die Kompetenzen der Gewerkschaften zu beschneiden, wird als Lösung für eine stagnierende Wirtschaftsentwicklung und hohe Arbeitslosenzahlen angepriesen. Die Vorwürfe, die sich gegen das Tarifvertragssystem richten, sind zwar seit der Bundestagswahl 2005 etwas leiser geworden (Bispinck/WSI-Tarifarchiv 2007); sie sind jedoch nur auf Eis gelegt. Dafür spricht etwa, dass die CDU in ihrem Entwurf für ein neues Grundsatzprogramm vom Mai dieses Jahres ihre Forderung nach gesetzlichen Öffnungsklauseln aktualisierte (CDU 2007, S. 48f.). In einem Minderheitsvotum sprach allerdings Peter Bofinger, Mitglied des Sachverständigenrats, den letzten Tarifabschlüssen, die nur geringe Lohnerhöhungen vorsahen, jede Ursächlichkeit für die positive Beschäftigungsentwicklung in den letzten zwei Jahren ab (SVR 2006, Ziff. 554). Wenn diese Ansicht breiter akzeptiert wäre, böte die Tarifautonomie keine Angriffsfläche: Die Gewerkschaften könnten tarifliche Lohnpolitik betreiben, ohne befürchten zu müssen, für die Entwicklungen am Arbeitsmarkt in die Verantwortung genommen zu werden. Die gegenteilige, u.a. vom Sachverständigenrat vertretene Auffassung (ebd., Ziff. 490f.) ist das Hauptargument für die Angriffe auf die Tarifautonomie: $\mathrm{Zu}$ hohe oder zumindest zu inflexible Löhne verhinderten ein markträumendes Gleichge- wicht auf dem Arbeitsmarkt. Ebenso wie staatliche Transferleistungen hätten Tariflöhne einen beschäftigungshemmenden Mindestlohneffekt (Lohnkompressionshypothese). Vor allem Frauen, Ältere, Geringqualifizierte und Jugendliche würden durch die Inflexibilität der Löhne vom Arbeitsmarkt ausgegrenzt. Von der Politik der Gewerkschaften profitiere daher nur eine kleine Gruppe von Arbeitnehmern, die männlichen, gut ausgebildeten der Kernaltersgruppe. Deren Besserstellung durch die Gewerkschaften erfolge daher auf Kosten der benachteiligten Gruppen. Das Recht dürfe die Tarifvertragsparteien nicht noch darin unterstützen, solche beschäftigungsschädlichen Verträge abzuschließen. Die wirtschaftlichen Untersuchungen, in denen auf den beschriebenen Effekt von Tariflöhnen hingewiesen wird, können allerdings nicht auf empirische Daten verweisen, sondern beruhen auf Plausibilitätserwägungen (Rose 2006, S. 80). Und ohnehin: Empirische Untersuchungen, z. B. zu den Ursachen von Lohnstarrheiten, stoßen auf grundsätzliche Schwierigkeiten (Franz/ Pfeiffer 2003, S. 25).

Der Beitrag zeigt, dass die rechtswissenschaftliche und die rechtspolitische Debatte um das Tarifrecht durch eine Überbetonung ökonomischer Logik gekennzeichnet ist. Anders ausgedrückt lautet die Ausgangsthese: In rechtspolitischen Erwägungen entscheiden sich Juristen implizit für einen Maßstab, anhand dessen sie die Gestaltung des Rechts beurteilen. Auch wenn es nicht immer beabsichtigt sein mag, die ökonomische Effizienz als entscheidenden Maßstab zugrunde zu legen, geschieht das in der rechtswissenschaftlichen Diskussion, indem den Argumenten der Wirtschaftswissenschaftler ein überproportional großes Ge- wicht eingeräumt wird und Untersuchungen anderer Wissenschaftsbereiche weit seltener zur Kenntnis genommen werden.

Im folgenden Abschnitt werden zunächst die Regelungen vorgestellt, die die Tarifautonomie rahmen und von denen fast alle Gegenstand von Änderungsvorschlägen sind. Auch wenn bereits wiederholt Gesetzesvorschläge ausformuliert wurden, ${ }^{1}$ hat es bisher keine Gesetzesänderungen in diesem Bereich gegeben. Wie das gesamte Arbeitsrecht ist das Tarifrecht aber stark durch Richterrecht geprägt, sodass sich eine Änderung der Rechtsprechung ebenso weitreichend auswirken kann wie eine Gesetzesänderung. Beachtung fand beispielsweise ein Urteil des Bundesarbeitsgerichts von 1999, in dem eine nachträgliche Genehmigung tarifwidriger betrieblicher Bündnisse für Arbeit durch die Gewerkschaften für zulässig gehalten wurde (BAG 1999a). ${ }^{2}$ Die Herangehensweise

\footnotetext{
1 Z. B. Entwurf der FDP von 2001 zur Sicherung betrieblicher Bündnisse für Arbeit, BT-Drs. 14/6548; Entwurf von CDU/CSU von 2003 für ein Arbeits rechtsmodernisierungsgesetz, BT-Drs. 15/1182.

2 Um Tarif- und sonstige Kollektivvereinbarungen mit ihren jeweils unterschiedlichen rechtlichen Voraussetzungen und Folgen unterscheiden zu können, müssten die Tarifparteien bei der Festlegung von Tarifstandards entscheidend an der Willensbildung beteiligt sein. Zumindest deren Unterrichtung vor Aufnahme der Verhandlungen sollte Bedingung für Kollektivvereinbarungen sein, die sich auf Tarifstandards auswirken (Zachert 2004a, S. 126).
}

Stefanie Kremer, Dr., ist Wissenschaftlerin im WSI in der Hans-Böckler-Stiftung, Projekt Regulierung des Arbeitsmarktes (REGAM). e-mail: stefanie-kremer@boeckler.de 
der Wirtschaftswissenschaft wird im dritten Abschnitt kurz vorgestellt. Im vierten Abschnitt wird anhand von Beispielen verdeutlicht, dass die Rechtswissenschaft teilweise Aussagen der Wirtschaftswissenschaften aufgreift, mit denen zugleich ökonomische Prämissen übernommen werden, die die juristische Methodik weitreichend überlagern. Der fünfte Abschnitt geht auf gesellschaftstheoretische Erklärungen der Tarifautonomie oder allgemein von kollektiv ausgeübter Autonomie ein. Schließlich wird angeregt, dass die Rechtswissenschaft mehr als bisher Ideen aus anderen Wissenschaftsbereichen als der Wirtschaftswissenschaft ziehen sollte.

\section{Der juristische Blick auf die Tarifautonomie}

Laut Bundesverfassungsgericht ist die Tarifautonomie ,darauf angelegt, die strukturelle Unterlegenheit der einzelnen Arbeitnehmer beim Abschluss von Arbeitsverträgen durch kollektives Handeln auszugleichen und damit ein annähernd gleichgewichtiges Aushandeln der Löhne und Arbeitsbedingungen zu ermöglichen " (BVerfG 1991, S. 229). Die Gewerkschaften werden als soziale Gegenspieler der Arbeitgeberverbände beschrieben; die Parteien würden antagonistische Interessen vertreten (Dieterich 2006, Rn. 18, 50). Mit dem Zusammenschluss zu Gewerkschaften hätten die Arbeitnehmer die Möglichkeiten der Selbsthilfe genutzt und mit den Mitteln des Arbeitskampfes kollektive Regelungen erzwungen (ebd.). Die Tarifautonomie wird seit einiger Zeit als Ausübung ,kollektiver Privatautonomie "verstanden. ${ }^{3}$

Art. 9 Abs. 3 Grundgesetz (GG) gewährleistet das Recht, zur Wahrung und Förderung der Arbeits- und Wirtschaftsbedingungen Vereinigungen zu bilden, und schützt damit die Tarifautonomie. Die gesetzlichen Regelungen und die Rechtsprechung müssen sicherstellen, dass dieses verfassungsrechtlich gewährleistete Recht wahrgenommen werden kann und die Tarifvertragsparteien die Arbeits- und Wirtschaftsbedingungen ihrer Mitglieder in kollektiven Verträgen autonom gestalten können. Im System der Verfassung ist die Tarifautonomie nicht Bestandteil der Verfahren demokratischer Legitimation, sondern ein Grundrecht. Sie ist eine positiv be- wehrte Freiheit. Das bedeutet, dass der Gesetzgeber die faktische Nutzung der Tarifautonomie ermöglicht, indem er sie durch Schutznormen ausgestaltet. Damit nutzt der Gesetzgeber eine der drei Möglichkeiten, den Handlungsspielraum eines Einzelnen zu vergrößern: indem er eine Kompetenz einräumt, Schutznormen erlässt oder eine Leistung im engeren Sinne gewährt (Alexy 1986, S. 222). Ein Recht, das in der Verfassung als bewehrte Freiheit konzipiert ist, darf weder von Privatpersonen noch vom Gesetzgeber (ungerechtfertigt) beschnitten werden, und eines, das als positiv bewehrte Freiheit einzuordnen ist, muss darüber hinaus vom Gesetzgeber so ausgestaltet werden, dass es faktisch genutzt werden kann (ebd., S. 203, 209f.). Der Gesetzgeber hat im Bereich der Tarifautonomie demnach eine Doppelfunktion: Er hat Normen aufgestellt, mit denen die Tarifautonomie ausgestaltet wurde, und andere, mit denen sie beschnitten wurde (Dieterich 2002, S. 10f.).

Regelungen des Tarifvertragsgesetzes (TVG) und des Betriebsverfassungsgesetzes (BetrVG) gestalten die Tarifautonomie aus. Sie sehen insbesondere vor:

- die nur durch das Günstigkeitsprinzip oder Öffnungsklauseln durchbrochene unmittelbare und zwingende Wirkung von Tarifnormen ( $\$ 4$ TVG); 4

- das Verbot, Betriebsvereinbarungen über Sachgebiete abzuschließen, die durch Tarifvertrag geregelt sind oder üblicherweise geregelt werden ( $\$ 77$ Abs. 3 BetrVG); - die Nachbindung von Tarifverträgen im Falle eines Austritts des Arbeitgebers aus dem Arbeitgeberverband bis zum Ende der Laufzeit des Tarifvertrages ( $\$ 3$ Abs. 3 TVG);

- die Nachwirkung von Tarifverträgen nach Ende der Laufzeit bis neue Arbeitsbedingungen aufgestellt werden ( $\$ 4$ Abs. 5 TVG) und

- die Möglichkeit des Bundesarbeitsministers, Tarifverträge für allgemeinverbindlich zu erklären ( $\$ 5 \mathrm{TVG})$.

Eine weitere Rahmung der Tarifautonomie, die jedoch nicht Gegenstand von Reformdiskussionen ist, ist die Feststellung des Bundesarbeitsgerichts, dass Arbeitskämpfe grundsätzlich rechtmäßig sind und die gegenseitigen Leistungspflichten während eines Arbeitskampfes ausgesetzt werden (BAG 1984). Die Abschaffung, Einschränkung oder Neuinterpretation der ge- nannten fünf gesetzlichen Regelungen wird mit dem Ziel diskutiert, beschäftigungsschädliche Tarifverträge zu vermeiden, und es wurde auch über neue Regelungen nachgedacht, z. B. den Gewerkschaften per Verfassungsänderung eine Pflicht aufzuerlegen, bei Tarifabschlüssen die beschäftigungspolitischen Auswirkungen zu beachten.

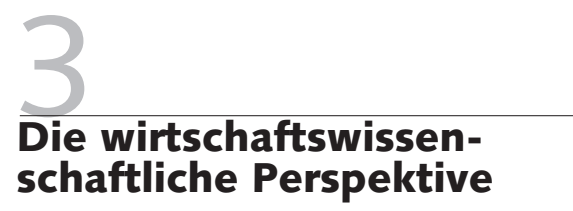

Wenn wirtschaftswissenschaftliche Studien gesetzliche Regelungen behandeln, betrachten sie sie unter ökonomischen Aspekten. Eine ökonomische Analyse des Rechts geht davon aus, dass jeder versucht, mit möglichst geringen Kosten den höchsten Nutzen zu erzielen, d.h. Recht wird unter dem Aspekt untersucht, wie es die Kosten bestimmter Handlungen beeinflusst: Regelungen sind entweder versteckte Preise oder Subventionen (Röhl 2005). Hierin enthalten ist die Annahme, dass jeder vorausschauend und in Kenntnis seiner Ressourcen handelt. Wirtschaftswissenschaftliche Untersuchungen zum Tarifvertragssystem richten sich auf die Kosten hoher Tariflöhne nicht nur für die Arbeitgeber, sondern - wie oben am Beispiel der Meinung des Sachverständigenrats beschrieben - auch für bestimmte Arbeitnehmergruppen. Diese Kosten gelten als mittelbare Kosten des Tarifrechts.

Ausgehend von diesen Prämissen konstatiert Werner Sesselmeier: Indem die Gewerkschaften die individuell abschöpfbaren Einkommenssteigerungen für die Insider, die mit hohem Humankapital ausge-

3 Übersichten zu dieser Diskussion bei Dieterich 2006, Rn. 54ff.; Kempen/Zachert (Zachert) § 4 Rn. 259. Lange galt überwiegend, dass die Tarifvertragsparteien staatlich legitimiert seien, da sie Aufgaben wahrnehmen würden, die andernfalls der Staat erfüllen müsste ("Delegationstheorie“). Der jetzt vorherrschenden "Theorie der kollektiven Privatautonomie" zufolge sind die Tarifvertragsparteien durch den Auftrag ihrer Mitglieder legitimiert.

4 §4 Abs. 3 TVG erlaubt Abweichungen von Tarifverträgen durch Individualarbeitsverträge oder Betriebsvereinbarungen nur, wenn die Tarifparteien dies in einer Öffnungsklausel gestattet haben oder wenn die Arbeitnehmer dadurch besser gestellt werden als nach dem Tarifvertrag. 
statteten, in der Regel männlichen und gut ausgebildeten Beschäftigten der Kernaltersgruppe erzielen, verstärken sie deren ohnehin gegenüber den Outsidern größere Verhandlungsmacht (Sesselmeier 2004, S. 129f.). Ein „neues Argument" entstehe durch den Zusammenschluss der Beschäftigten zu Gewerkschaften nicht, sondern diese verstärkten nur das ohnehin bestehende Machtgefälle zwischen Insidern und Outsidern (ebd.). Warum die Tätigkeit der Gewerkschaften gesetzlich gerahmt und damit gefördert wird, sei durch einen beiderseitigen Vorteil für Gewerkschaften und Staat zu erklären: Das Tarifrecht sei den Gewerkschaften im Rahmen eines „politischen Tausches“ vom Staat als Gegenleistung dafür gewährt worden, dass die Arbeit stärker besteuert ist als das Kapital (ebd.).

Ebenfalls um mögliche Kosten des Tarifvertragssystems zu ermitteln, vergleicht Ronald Schettkat es mit der Unternehmensberatung Hay-Consulting, die ein Informationspool für außertarifliche Managementgehälter betreibt (Schettkat 2003, S. 272f.). In ähnlicher Weise informierten die Tarifvertragsparteien im Bereich normaler Angestellter über die in der Region und Branche gezahlten Gehälter. Die Tarifvertragsparteien wirkten wie ein Auktionator, der für einen Ausgleich von Angebot und Nachfrage sorgt: Die Gewerkschaften bündelten die Angebotsseite, die Arbeitgeberverbände die Nachfrageseite. Zudem delegierten kollektive Lohnverhandlungen Einkommenskonflikte an professionelle Unterhändler und beseitigten eine innerbetriebliche Konfliktquelle. Bereits diese beiden Beispiele machen deutlich, dass die Funktionsbeschreibungen der Wirtschaftswissenschaft deutlich von der juristischen abweichen. ${ }^{5}$

\section{4 \\ Die Ökonomie in der juristischen Tarifrecht- Debatte}

Die wirtschaftswissenschaftlichen Beschreibungen der Rolle der Gewerkschaften und der Funktion des Tarifrechts klingen für Rechtswissenschaftler nicht nur teilweise sonderbar - einige sind kaum vereinbar mit der juristischen Sicht. Umso erstaunlicher ist es, dass sie dennoch in der juristischen Debatte so viel Unruhe stiften. Zwar sprechen gewichtige rechtliche Gründe gegen die ökonomisch motivierten Änderungsvorschläge und einige Rechtswissenschaftler machen diese auch geltend. Andere räumen den wirtschaftswissenschaftlichen Vorschlägen jedoch einen sehr großen Spielraum ein, mit teilweise bedenklichen Folgen.

\subsection{DEUTUNGSDIFFERENZEN}

Die Feststellung Sesselmeiers, dass durch den Zusammenschluss der Arbeitnehmer zu Gewerkschaften kein neues Argument entstehe, sondern nur bestehende Arbeitsmarktmechanismen verstärkt werden, steht in einem deutlichen Widerspruch zu der juristischen Wahrnehmung. Ihr zufolge haben die Arbeitnehmer durch den $\mathrm{Zu}-$ sammenschluss zu Gewerkschaften die Möglichkeiten der Selbsthilfe genutzt, um kollektive Regelungen zu erzwingen. Ebenfalls anders als in der juristischen Sicht erscheinen die Gewerkschaften bei Schettkat nicht in erster Linie als soziale Gegenspieler der Arbeitgeberverbände, sondern sie stellen sich zusammen mit diesen als nützliche Dienstleister dar. Die Tarifvertragsparteien werden mit einer Unternehmensberatung verglichen, zudem wirkten sie befriedend auf die Betriebe. Diese Beschreibungen sind schwer zu vereinbaren mit der rechtswissenschaftlichen Sicht, die die Tarifvertragsparteien als soziale Gegenspieler wahrnimmt, die antagonistische Interessen vertreten. Zwar können Tarifverträge im Ergebnis eine ,win-win-Situation“ "herstellen (Oppolzer/Zachert 2000, S. 231) und sich als vorteilhaft sowohl für Beschäftigte als auch Unternehmen erweisen. Wie bei jedem Vertrag muss es den beteiligten Parteien aber möglich sein, bei sich zu bleiben, also ausschließlich aus der Perspektive zu entscheiden, die sich aus der Verfolgung eigener Interessen ergibt. Die Vertragsform ist ihrer Herkunft nach privatrechtlich, und die Vertragsparteien müssen die Möglichkeit haben, Vorteile für den Vertragspartner außer Acht zu lassen oder höchstens strategisch zu berücksichtigen. Die Betonung der allgemeinen Nützlichkeit des Tarifvertragssystems ist sinnvoll als Hinweis an den Gesetzgeber, um ihn von einer in Betracht gezogenen Einschränkung der Tarifautonomie abzuhalten. Nebenwirkung ist aber, dass der Gesetzgeber sich berechtigt sieht, den Fortbestand der Tarifautonomie von der anhaltenden Nützlichkeit des Tarifvertragswesens abhängig zu machen. Er geht hiermit den Schritt von der Feststellung, dass das Tarifvertragssystem der Allgemeinheit, einschließlich den Unternehmen, nützt, zu der normativen Festlegung, dass es das auch tun muss. Wiederholt hat der Gesetzgeber in diesem Sinne an die Tarifvertragsparteien appelliert, in ihrem langfristigen Interesse am Systemerhalt bestimmte, nämlich "gemeinnützige“ Tarifverträge abzuschließen. Das Typische vertraglicher Vereinbarungen, dass das Entstehen einer „win-win“-Sitution von keinem der Beteiligten beabsichtigt werden muss, geht damit verloren.

\subsection{AUSHÖHLUNG RECHTLICHER GRUNDSÄTZE}

Unabhängig von ihrer wirtschaftlichen Nützlichkeit ist die Daseinsberechtigung der Tarifvertragsparteien zwar durch die Verfassung gesichert. Die Feststellung, dass Änderungen im kollektiven Arbeitsrecht, die die Existenz des Tarifvertragssystems gefährden, verfassungswidrig sind, müssen aber Juristen treffen. Dazu kommt es nicht, wenn sie wirtschaftlichen Argumenten so großes Gewicht einräumen, dass sie die Garantie der Verfassung überlagern, was in den folgenden zwei Beispielen der Fall ist: Der Jurist Eduard Picker beschreibt den Vorrang ökonomischer Argumente ohne Umschweife mit der Feststellung, selbst die Grundgesetze der Staaten müssten über kurz oder lang den Grundgesetzen der Ökonomie folgen (Picker 2002, S. 23f.). Alles Recht, insbesondere alle Rechtsinstitute seien zeitgebunden, und der Koalitionsschutz sei rein geschichtlich bedingt. Heute seien die Interessenverbände nicht mehr gefährdet, weshalb deren Schutz relativiert werden müsse. Mit der Feststellung, dass die Interessenverbände nicht mehr gefährdet seien, wird eine These aufgegriffen, die auch von anderen Juristen und auch im Zusammenhang mit Diskussionen über das Individualarbeitsrecht vertreten wird. Sie besagt, dass die Arbeitswelt nicht mehr durch Über-/Unterordnungsverhältnisse, sondern durch Kooperation geprägt sei;

\footnotetext{
5 Mit diesen zwei Beispielen ist hier nur eine grobe Annäherung an das Thema möglich. Den Eingang wirtschaftlicher Aspekte in die juristische Diskussion um das Günstigkeitsprinzip beschreibt Rehder (2006). Allgemein zu Überschneidungen zwischen ökonomischer und juristischer Argumentation im Arbeitsrecht Kremer (2007).
} 
daher sei zweifelhaft, dass es noch eine gestörte Vertragsparität gebe (so z. B. Reichold 2002, S. 276). Was in Pickers Argumentation nicht zur Sprache kommt, ist, dass die „Grundgesetze der Ökonomie“ im Unterschied zu den „Grundgesetzen der Staaten" nicht demokratisch legitimiert sind, sondern nur Erklärungen für wirtschaftliche Phänomene anbieten.

Ein weiterer Jurist, Volker Rieble, machte neben einigen anderen Vorschlägen zur Änderung des Tarifvertragsrechts auch den, die zwingende Wirkung von Tarifverträgen abzuschaffen (Rieble 2004). Der Meinungsstreit um das Günstigkeitsprinzip, das die zwingende Wirkung durchbricht, indem es Abweichungen von Tarifverträgen zugunsten des Arbeitnehmers erlaubt (vgl. Fn. 4), behandelt überwiegend die Frage, was als „Besserstellung “ eines Arbeitnehmers zu bewerten sei bzw. welche Regelungen bei der Beurteilung berücksichtigt werden dürfen. Das Bundesarbeitsgericht ist bisher entgegen immer lauter werdender Kritik bei seiner Meinung geblieben, dass eine Arbeitszeitverlängerung oder eine Lohnkürzung im Tausch gegen eine befristete Beschäftigungsgarantie keine Besserstellung sei (grundlegend BAG 1999b). Die betrieblichen Bündnisse für Arbeit sind daher häufig rechtswidrig was allerdings so lange nicht zum Tragen kommt, bis eine Gewerkschaft dagegen klagt, was keineswegs bei jeder rechtswidrigen betrieblichen Vereinbarung der Fall ist. Wenn dem insbesondere von Rieble gemachten Vorschlag gefolgt würde, die zwingende Wirkung von Tarifverträgen abzuschaffen, würde überhaupt keine gerichtliche Prüfung mehr stattfinden, ob eine Regelung einen Arbeitnehmer besserstellt. $\mathrm{Zu}$ befürchten ist bei einer Aufhebung oder Einschränkung der zwingenden Wirkung, dass Tarifverträge zu unverbindlichen Leitlinien würden und das Tarifvertragssystem auf Dauer funktionsuntüchtig würde, was mit der verfassungsrechtlichen Garantie nicht zu vereinbaren wäre (Dieterich 2002). Zwar ist im angloamerikanischen Rechtskreis auch ein Tarifvertragssystem funktionstüchtig, das keine rechtlich verbindlichen Vereinbarungen kennt, jedoch finden sich in diesem Rechtskreis auch andere Strukturprinzipien, die einen direkten Vergleich einzelner Elemente verbieten (Zachert 2004a, S. 123). Ohne weitere Begründung stellt Rieble jedoch fest, dass diese Einschränkung der Tarifautonomie wegen der damit erzielbaren beschäfti- gungspolitischen Wirkung verhältnismäßig sei. Die Verhältnismäßigkeit aber ist eine Leitregel allen staatlichen Handelns, die aus dem Rechtsstaatsprinzip (Art. 20 Abs. 3 GG) und den Freiheitsgrundrechten abgeleitet wird, wonach eine staatliche Maßnahme, z. B. eine Gesetzesänderung, im Hinblick auf den verfolgten Zweck nicht über das erforderliche und geeignete $\mathrm{Ma}$ hinaus unangemessen in Rechtspositionen des Bürgers eingreifen darf. Merkmal einer Abwägung im Rahmen der Verhältnismäßigkeitsprüfung wäre daher eine Begründung, warum ein Recht im konkreten Fall hinter einem anderen zurücksteht. Im juristischen Kontext enthält der begründungslose Hinweis auf die Verhältnismäßigkeit einer Abschaffung der zwingenden Wirkung daher die implizite Aussage, dass die Tarifautonomie bei einer Abwägung mit dem Ziel der Beschäftigungsförderung nichts wiegt, was nicht zutreffend sein kann. Diese Aussage kann nur getroffen werden, wenn man die ökonomische Perspektive übernimmt, die die Frage der Verfassungsmäßigkeit nicht kennt, sondern fragt, wie sich mit möglichst geringen Kosten der größte Nutzen erzielen lässt.

\subsection{ZWISCHENFAZIT}

Mit dem ökonomischen Blickwinkel scheint auch das Rechtsverständnis der Wirtschaftswissenschaften Eingang in die Rechtswissenschaft gefunden zu haben: Alles, was die Wirtschaftstätigkeit rechtlich begrenzt, erscheint als ein von außen kommendes Hindernis, das spontane Abläufe in künstliche Bahnen lenkt. Dieses Rechtsverständnis setzt private Handlungsfreiheit mit einem möglichst großen Wahlrecht gleich und übersieht, dass privatautonomes wirtschaftliches Handeln nie voraussetzungslos ist. Es ist z. B. immer von einer militärischen Sicherung und einer Steuergesetzgebung gerahmt (Habermas 1992, S. 189). Dieses Verständnis des Rechts widerspricht auch der Charakterisierung der Tarifautonomie, die sich im System der Grundrechte als eine positiv bewehrte Freiheit darstellt. Der Gesetzgeber würde gerade dann die Freiheit des Einzelnen beeinträchtigen, wenn er den verfassungsrechtlichen Auftrag ignorieren würde, sie in bestimmten Bereichen gesetzlich zu rahmen. ${ }^{6}$ In den Argumentationen Riebles und Pickers findet die Unterscheidung zwischen ausgestaltenden und beschneidenden Normen im Bereich des Tarifrechts aber keine Berücksichtigung. Gleiches gilt für die besonderen formalen Anforderungen der Verhältnismäßigkeitsprüfung oder die Rechtsprechung des Bundesverfassungsgerichts zur Funktion der Tarifautonomie. Wirtschaftswissenschaftliche Argumentationsmuster haben hier die Eigenarten juristischer Argumentation verdrängt. ${ }^{7}$

\section{Gesellschaftstheoretische Konzeptionen kollektiver Autonomie}

"Gesellschaftstheorie“ ist kein feststehender Begriff. Nach der weiten Definition, die wikipedia anbietet, sind Gesellschaftstheorien ein Teilbereich der Soziologie, die versucht, den Aufbau und die Entwicklung der Gesellschaft zu beschreiben und zu erklären. Geläufiger als der Begriff „Gesellschaftstheorie" im Deutschen ist der Begriff „social theory“ im Englischen. Laut wikipedia deckt er das Themengebiet ab, mit dem vorab die Soziologie umschrieben wurde. Der Begriff umfasst ein „theoretical framework" und Untersuchungen eines interdisziplinären Felds: Sowohl aus der Anthropologie, der Ökonomie, der Geschichtswissenschaft, der Literaturtheorie, der Philosophie, der Soziologie und der Theologie würden Ideen aufgegriffen. Solche Untersuchungen der Soziologie oder der „social theory", die den Anspruch haben, mit Hilfe eines theoretischen Rahmens den Aufbau der Gesellschaft zu erklären, spielen bei der Debatte um eine Reform des Tarifrechts bisher leider kaum eine Rolle. Sogar in der Diskussion um das richtige Verhältnis zwischen individueller und kollektiver (Privat-)Autonomie, die in den letzen Jahren geführt wurde (vgl. Fn. 3), werden sol-

\footnotetext{
6 Dieses Rechtsverständnis teilte auch Max Weber, dem zufolge es Aufgabe des Rechts ist, einen rein formalen Rahmen aufzustellen (Weber 1964, S. $160 \mathrm{ff}$.). Nicht das demokratische Verfahren, sondern allein die grammatische Form der Gesetze erzeugt demzufolge deren Legitimität. Die Ei genheiten des Rechts im Sozialstaat wurden von Weber jedoch falsch eingeschätzt, und er hat auch versäumt, sich einer soziologischen Rechtswissenschaft zuzuwenden (Zachert 2004b, S. 3f.).

7 Abgesehen von diesen methodischen begegnet es auch grundsätzlichen Bedenken, bei der Beurteilung des Rechts ökonomische Kriterien absolut zu setzen (Kunz/Mona 2006, S. 234ff. m.w. N.), auf die hier nicht näher eingegangen werden kann.
} 
che Untersuchungen eher selten aufgegriffen, obwohl dieses Verhältnis kaum anders als unter Verwendung von Theorien zum gesellschaftlichen Aufbau und von soziologischen Befunden näher beschrieben werden kann.

Die im Folgenden beispielhaft vorgestellten Argumentationen von Jürgen $\mathrm{Ha}$ bermas, Axel Honneth und Michel Foucault beschreiben prinzipielle Anforderungen an das Recht, die erfüllt sein müssen, wenn es Ausdruck individueller Autonomie sein soll. Habermas kann in der Tradition klassischer Gerechtigkeitstheorien verortet werden und steht hier stellvertretend für diese. Andere herausragende zeitgenössische Vertreter wie etwa John Rawls wären hier ebenfalls zu behandeln, worauf jedoch aus Platzgründen verzichtet werden muss. Auf Bereiche wie die Systemtheorie oder die um den Begriff des Risikos kreisenden Theorien kann hier ebenfalls nicht eingegangen werden. Die Ansätze von Honneth und Foucault in einer Untersuchung zur Tarifautonomie heranzuziehen, bietet sich an, weil die Ausübung von Selbstbestimmung durch Kollektive bei ihnen besonderes Gewicht einnimmt. Honneth zählt wie Habermas zur Zweiten Generation der Kritischen Theorie. Das Denken Foucaults ist von der Auseinandersetzung mit dem französischen Strukturalismus geprägt, Gemeinsamkeiten mit den Post-Strukturalisten sind aber begrenzt. ${ }^{8}$

\subsection{HABERMAS: VORAUSSETZUNGEN FÜR DEMOKRATIE}

Eine kollektive Regelung, die nicht in einer Weise zustande gekommen ist, dass sie es den Betroffenen erlaubt, sich als ihr Autor zu begreifen, ist Habermas zufolge illegitim. Eine legitime Regelung hingegen müsse den Betroffenen ermöglichen, zwischen zweierlei Umgangsweisen zu wählen: Zwischen der Möglichkeit, mit dem von einer Regelung ausgehenden Zwang strategisch umzugehen, sie als eine "soziale Tatsache“ zu betrachten, und der Möglichkeit, die Regelung als in einem höheren Interesse gerechtfertigt anzusehen und in eigene Motive zu übersetzen (Habermas 1992, S. 44ff., 91, 154). Soziale Integration erfordere demnach, dass moralische Diskurse, d.h. "anspruchsvolle Kommunikationsformen“" einer demokratischen Meinungs- und Willensbildung, rechtlich institutionalisiert werden (ebd., S. 235ff., 361). Habermas selbst hat zwar seine Aufmerksamkeit nur am Rande auf das Tarifvertragssystem gerichtet, es zumindest nicht unter den beschriebenen Gesichtspunkten beurteilt. Dies bietet sich jedoch durchaus an. Im Tarifvertragssystem könnte ein Beitrag zur staatlichen Legitimation gesehen werden, der es den Individuen erleichtert, die verbindlichen Regelungen des Staates in eigene Motive zu übersetzen. In diesem Sinne findet sich beispielsweise in der Rechtswissenschaft die Feststellung, dass es bei der Koalitionsfreiheit um die menschliche Freiheit gehe, die eine Zentralvoraussetzung jeder demokratischen Verfassungsordnung ist (Kempen/Zachert (Kempen) 2006, Grundlagen, $\$$ 63). Im Einzelnen könnte das Tarifvertragssystem als Ausgleich für jene Regelungen verstanden werden, die Besitzstände schützen. So könnte es auch jenen, die nicht über Vermögen oder Kapital verfügen, ermöglichen, die staatlichen Regelungen als ihre zu verstehen.

\subsection{HONNETH: REGELUNG VON ANERKENNUNG}

Honneth kommt auf einem Umweg zu Fragen der Legitimation des Rechtssystems. Sein Interesse richtet sich auf die Funktion nicht ausdrücklich zum Handeln ermächtigter Kollektive für politische Prozesse und auf deren Beweggründe. Er sieht in ihnen Träger des gesellschaftlichen Fortschritts. Sie könnten Auskunft über moralisches Unbehagen geben, indem sie einen Geltungsüberhang einklagen, den die gesellschaftliche Ordnung besitzt und dem die tatsächlichen Anerkennungsverhältnisse widersprechen. Nur was den Organisationsgrad politischer Bewegungen angenommen hat, werde als moralisch ernst zu nehmendes Problem registriert, denn im Unterschied zu einzelnen Personen hätten soziale Bewegungen einen ausreichenden Publizitätsgrad, um Beachtung zu finden (Honneth 2003, S. 134, 141). In diesem Sinne sei auch die Arbeiterbewegung ein Kampf um eine gerechte Verteilung von Anerkennung gewesen, nämlich um Bewertungsmaßstäbe, die die gesellschaftlichen Beiträge jedes einzelnen gleichmäßig wertschätzen (ebd., S. 183). Soziale Integration müsse immer als ein Prozess der Inklusion durch geregelte Formen der Anerkennung gesehen werden; nur dann werde eine Gesellschaft von ihren Mitgliedern als legitimes Ordnungsgefüge wahrgenommen (ebd., S. 205). Allerdings sieht Hon- neth weder die Arbeiterbewegung noch die Tarifvertragsparteien als einen geeigneten Seismografen für moralisches Unbehagen. Die Arbeiterbewegung werde inzwischen nicht mehr wahrgenommen von jenen, die die normativen Ansprüche sozialer Bewegungen untersuchen und auf eine Umsetzung ihrer Zielsetzungen hinwirken; stattdessen richte sich die Aufmerksamkeit auf Aktionsgruppen und Protestbewegungen, die unter dem Sammelbegriff „neue soziale Bewegungen" behandelt würden (ebd., S. 136). ${ }^{9}$ Honneth stellt damit strenge Anforderungen an jene kollektiven Akteure, die moralische Kriterien in den Prozess staatlicher Legitimation einbringen können. Grundsätzlich wäre jedoch nicht ausgeschlossen, das Tarifvertragssystem, genauer gesagt die Gewerkschaftstätigkeit, als Beitrag zur moralischen Akzeptanz des Rechtssystems zu sehen. Die Gewerkschaften könnten grundsätzlich als Kollektive verstanden werden, die darauf hinweisen, dass die tatsächlichen Anerkennungsverhältnisse den von der geltenden Ordnung behaupteten widersprechen.

\subsection{FOUCAULT: STREUUNG POLITISCHER ENTSCHEIDUNGEN}

Foucault formulierte keine konkreten Anforderungen an ein legitimes Rechtssystem, drückte aber die Hoffnung auf die Entstehung eines "neuen Rechts“ aus, das vom Prinzip der Souveränität befreit sein würde (Foucault 1999, S. 50). Dass die Idee der Souveränität zum Selbstverständnis staatlicher Machtausübung gehöre, sah er als ein Überbleibsel der Monarchie, das es unmöglich mache, sich den Staat anders denn als Handlungssubjekt nach dem Vorbild eines Individuums vorzustellen (Foucault 1983). ${ }^{10}$ Ein „,neues Recht“ entstehe, indem es von Privatpersonen (kollektiv) in An-

\footnotetext{
8 Überblicke über in der Nähe der Rechtsphilosophie und -soziologie angesiedelte Theorien finden sich bei Rottleuthner (1987); Buckel et al. (2006); Kunz/Mona (2006).

9 In Tarifauseinandersetzungen würden, ebenso wie in der Steuerpolitik, Verteilungsfragen auf staatlicher Ebene behandelt (Habermas 1992, S. 179).

10 In der von einigen Rechtswissenschaftlern vertretenen Auffassung, dass individuelle und kollektive Freiheiten gleichrangig sind und miteinander konkurrieren anstatt die kollektiven als Schutz und Fortsetzung der individuellen zu sehen, findet sich dieses Rechtsverständnis widergespiegelt (weiterführend Kempen/Zachert (Zachert), § 4 Rn. 258ff.).
} 
spruch genommen werde, die von niemandem beauftragt sind, die nur das Bedürfnis teilen, sich gegen bestimmte Ereignisse zu wenden (nach Lemke 2001, S. 270f.). Foucault hebt hervor, dass bereits mit dem kollektiven Handeln ein „Recht“ entstehe, dem der gleiche Stellenwert zuzugestehen sei wie dem staatlichen Recht. Da das „neue Recht" sich auch gegen eine staatliche Regierung richten soll, sofern sie politische Entscheidungsprozesse monopolisiert, wird die Unvermeidbarkeit von Konflikten betont. Nicht autorisierte soziale Bewegungen spielen demnach auch bei Foucault eine entscheidende Rolle. Gerade im Hinblick auf seine Geschichte ließe sich das Tarifvertragssystem als Versuch der Etablierung eines ,neuen Rechts“verstehen, das ohne das Prinzip der Souveränität auskommt. In der Tatsache, dass die Tarifvertragsvereinbarungen die unmittelbare und zwingende Wirkung mit staatlichen Gesetzen gemeinsam haben, lässt sich auch der Gedanke wiederfinden, dass dem aus dem kollektiven Handeln entstehenden „Recht“ der gleiche Stellenwert zugestanden werden müsse wie dem staatlichen.

Die drei Beispiele verdeutlichen, dass bei einem Blick auf die gesellschaftliche Struktur die Bedeutung des Tarifvertragssystems zu Tage tritt und auch begründet werden kann. Wenn über rechtliche Änderungen bei der Tarifautonomie nachgedacht wird, sollten auch diese Erwägungen Berücksichtigung finden.

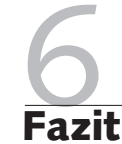

Insbesondere aus den soziologischen und philosophischen Erklärungen zum Aufbau der Gesellschaft lassen sich Argumente für die Bedeutung des Tarifvertragssystems ziehen. Dies machen die dargestellten Ansätze deutlich. Die gesellschaftstheoretischen Arbeiten sollten allerdings nicht als Korrektiv der Wirtschaftswissenschaften benutzt werden, sondern ihre eigenständi- ge Berechtigung muss unzweifelhaft bleiben. Zwar sieht die Rechtswissenschaft die Tarifautonomie seit Kurzem wieder als Ausübung „kollektiver Privatautonomie“, womit sie sich einigen der beschriebenen theoretischen Argumentationen durchaus annähert. Beispielsweise ist eine „kollektive Privatautonomie" näher mit einem vom Prinzip der Souveränität befreiten „neuen Recht" im Sinne Foucaults verwandt als eine Tarifautonomie, die als Ausprägung staatlicher Kompetenzen verstanden wird, wie es früher der Fall war. Dennoch spielen wirtschaftswissenschaftliche Argumente in der Rechtswissenschaft eine deutlich übergewichtige Rolle. Sie haben eine „Diskurskoalition“ zwischen Ökonomie und Rechtswissenschaft bewirkt (Rehder 2006), die die Tarifautonomie in Rechtfertigungsnot gebracht haben. Der Blick auf die gesellschaftlichen Strukturen und die Voraussetzungen sozialer Integration lässt demgegenüber keinen Rechtfertigungsbedarf des Tarifvertragssystems erkennen. 
Alexy, R. (1986): Theorie der Grundrechte, Frankfurt a. M. Bispinck, R./WSI-Tarifarchiv (2007): Tarifpolitischer Jahresbericht 2006: Aufwärtstrend bei Abschlüssen - Konflikte um Beschäftigungssicherung und Tarifstandards, in: WSI-Mitteilungen 2, S. 55-62

Buckel, S./Christensen, R./Fischer-Lescano, A. (2006): Neue Theorien des Rechts, Bern/Stuttgart/Wien

CDU (2007): Grundsätze für Deutschland. Beschluss der Grundsatzprogramm-Kommission der CDU Deutschlands vom 7.Mai http://www.grundsatzprogramm.cdu.de/doc/070507-grundsatzprogramm-kommission-entwurf-2.pdf

Dieterich, T. (2002): Flexibilisiertes Tarifrecht und Grundgesetz, in: Recht der Arbeit 1, S. 1-17

Dieterich, T. (2006): Art. 9 Grundgesetz, in: Dieterich, T./Müller-Glöge, R. /Preis, U./Schaub, G.: Erfurter Kommentar zum Arbeitsrecht, 6. Aufl., München

Foucault, M. (1983): Der Wille zum Wissen. Bd. 1, Frankfurt a. M. Foucault, M. (1999): In Verteidigung der Gesellschaft. Vorlesungen am Collège de France, Frankfurt a. M.

Franz, W./Pfeiffer, F. (2003): Zur ökonomischen Rationalität von Lohnrigiditäten aus der Sicht von Unternehmen, in: Jahrbuch für Nationalökonomie und Statistik 223, S. 23-43

Habermas, J. (1992): Faktizität und Geltung, Beiträge zur Diskurstheorie des Rechts und des demokratischen Rechtstaats, Frankfurt a. M.

Honneth, A. (2003): Umverteilung als Anerkennung. Eine Erwiderung auf Nancy Fraser, in: Honneth, A./Fraser, N.: Umverteilung oder Anerkennung? Eine politisch-philosophische Kontroverse, Frankfurt a. M., S. 129-224

Kempen, O. E./Zachert, U. (2006): TVG. Tarifvertragsgesetz, 4. Aufl., Frankfurt a. M.

Kremer, S. (2007): Bewusstseins- und Verhaltensänderungen durch die „Flexibilisierung“ des Arbeitsrechts. Eine Gouvernementalitäts-Studie, Baden-Baden (im Erscheinen)

Kunz, K.-L./Mona, M. (2006): Rechtsphilosophie, Rechtstheorie, Rechtssoziologie, Bern/Stuttgart/Wien

Larenz, K. (1992): Methodenlehre der Rechtswissenschaft, verkürzte Studienausgabe, 2. Aufl., Berlin/Heidelberg

Lemke, T. (2001): Freiheit ist die Garantie der Freiheit. Michel Foucault und die Menschenrechte, in: Vorgänge 40, S. 270-276

Oppolzer, A./Zachert, U. (2000): Zur Zukunft des Tarifvertrages: Versuch einer Synthese, in: Dies.: Krise und Zukunft des Flächentarifvertrages, Baden-Baden, S. 215-231
Picker, E. (2002): Tarifautonomie - Betriebsautonomie - Privatautonomie. Zum Legitimationsgrund der arbeitsrechtlichen Regelungsmächte, in: Rüthers, B. (Hrsg.): Der Konflikt zwischen Kollektivautonomie und Privatautonomie im Arbeitsleben, Baden-Baden, S. 11-32

Rawls, J. (2003): Eine Theorie der Gerechtigkeit, Frankfurt a. M. Reichold, H. (2002): Arbeitsrecht - Lernbuch nach Anspruchsgrundlagen, München

Rieble, U. (2004): Tarifvertrag und Beschäftigung, in: Zeitschrift für Arbeitsrecht 1, S. 1- 65

Rehder, B. (2006): Recht und Politik beim Wandel des deutschen Flächentarifs. Juristen als politische Akteure im System der Arbeitsbeziehungen, in: Politische Vierteljahresschrift 2, S. 169-192

Röhl, K. (2005): Recht und Wirtschaft als Thema der Rechtssoziologie, in: Zeitschrift für Rechtssoziologie 1, S. 3-34

Rose, E. (2006): Tarifautonomie: Perspektiven und Alternativen. Das Recht der tariflichen Entgeltfindung konfrontiert mit den Herausforderungen der globalen Informationsgesellschaft, Baden-Baden Rottleuthner, H. (1987): Einführung in die Rechtssoziologie, Darmstadt Schettkat, R. (2003): Reformen in Deutschland: zu wenig, zu spät? WSIMitteilungen 5, S. $267-274$

Sachverständigenrat zur Begutachtung der gesamtwirtschaftlichen Entwicklung (SVR) (2006): Jahresgutachten 2006/2007. Widerstreitende Interessen - ungenutzte Chancen, November Sesselmeier, W. (2004): Deregulierung und Reregulierung der Arbeitsmärkte im Lichte der Insider-Outsider-Theorie, WSI-Mitteilungen 3, S. 125-131

Zachert, U. (2004a): Tarifvertrag, Günstigkeitsprinzip und Verfassungsrecht, in: Arbeit und Recht 4, S. 121-129

Zachert, U. (2004b): Legitimation arbeitsrechtlicher Regelungen aus historischer und aktueller Sicht, in: Recht der Arbeit 1, S. 1-8

\section{Rechtsprechung}

Bundesarbeitsgericht (BAG) (1984): Urteil vom 12.9.1984, in: Neue Juristische Wochenschrift 1985, S. 2515

Bundesarbeitsgericht (BAG) (1999a): Urteil vom 20.4.1999, in: Neue Zeitschrift für Arbeitsrecht 19, S. 1059-1064

Bundesarbeitsgericht (BAG) (1999b): Beschluss vom 20.4.1999, in: Neue Zeitschrift für Arbeitsrecht 16, S. 887-894

Bundesverfassungsgericht (BVerfG) (1991): Beschluss vom 26.6.1991, in: Entscheidungssammlung (BVerfGE) 84, S. 212-232 